\title{
A COMPARISION STUDY OF TIME DOMAIN EQUALIZATION TECHNIQUE USING ULTRAWIDE BAND RECEIVERS PERFORMANCE FOR HIGH DATA RATE WPAN SYSTEM
}

\author{
Susmita Das, Member, IEEE* and Bikramaditya Das* \\ *Department of Electrical Engineering, National Institute of Technology, \\ Rourkela -769008, India \\ sdas@itrkl.ac.in \\ adibik09@gmail.com
}

\begin{abstract}
For high data rate ultra wideband communication system, performance comparison of Rake, MMSE and Rake-MMSE receivers is attempted in this paper. We have taken into account of impact of all the parameters such as the effect of the number of Rake fingers and equalizer taps on the error performance by combating ISI are the major focus of our research. The bit error rate performances are investigated using semi analytical approach and Monte-Carlo simulation on IEEE 802.15.3a UWB channel models. A detail study on UWB channel model (IEEE 802.15.3a) is carried out using four channel models CM1CM4. Simulation results show that the bit error rate probability of rake-mmse receiver is much better than Rake receiver and MMSE equalizer. Within the distance 0 to 4 meter Rake-MMSE performs better in CM1 (LOS) channel model than that of CM2 (NLOS) channel model. Further the simulation results on non-line of sight indoor channel models illustrate bit error rate performance of Rake-MMSE improves for CM3 model with smaller spread compared to CM4 channel model. We show that for a MMSE equalizer operating at low to medium SNR's, the number of Rake fingers is the dominant factor to improve system performance, while at high SNR's the number of equalizer taps plays a more significant role in reducing error rates.
\end{abstract}

KEYWORDS

UWB, Rake receiver, MMSE, Rake-MMSE, Bit Error Rate.

\section{INTRODUCTION}

Ultra Wideband Radio (UWB) is an emerging technology with big promise in imaging systems, vehicular radar systems, and communication and measurement systems. UWB technology has for many years been used in radar and military communications but has not been allowed on the open market prior to 2002. In April 2002, the federal communication commission (FCC) lifted the restriction on the use of UWB technology for non-military applications. Since then, more industries have started developing UWB systems. UWB systems send information with extremely short duration pulses, therefore allowing high speed data communication and diversity against multipath. Several attempts are being made to use UWB at the physical layer for personal area networks (PAN) to meet the FCC standards. FCC has released $3.1 \mathrm{GHz}$ to 10.6 $\mathrm{GHz}$ frequency spectrum with a restriction on minimum transmission bandwidth of $500 \mathrm{MHz}$. In order to reduce interference with existing narrowband communication systems the maximum transmit power spectral density of UWB is restricted to $-41.3 \mathrm{dBm} / \mathrm{MHz}$, which restricts the use of UWB to PAN. Ultra-wideband (UWB) has recently evoked great interest and its potential 
strength lies in its use of extremely wide transmission bandwidth. Furthermore, UWB is emerging as a solution for the IEEE 802.15a (TG3a) standard which is to provide a low complexity, low cost, low power consumption and high data-rate among Wireless Personal Area Network (WPAN) devices. An aspect of UWB transmission is to combat multipath propagation effects. Rake receivers can be employed since they are able to provide multipath diversity [1-3]. Another aspect is to eliminate or combat the inter-symbol interference (ISI) which distorts the transmitted signal and causes bit errors at the receiver, especially when the transmission data rate is very high as well as for which are not well synchronized. In [1,3,4], the "rake decorrelating effect" was mentioned as a way to combat ISI. Combination of spatial diversity combining and equalization is a well established scheme for frequency selective fading channels [10]. In [5], a combined rake and equalizer structure was proposed for high data rate UWB systems. In this paper, the performance of a rake-MMSE-equalizer receiver similar to [5] is investigated for different number of rake fingers and equalizer taps using a semi-analytical approach. We propose at first to study time equalization with combined Rake-mmse equalizer structure. We show that, for a MMSE equalizer [6] operating at low to medium SNR's, the number of Rake fingers is the dominant factor to improve system performance, while, at high SNR's the number of equalizer taps plays a more significant role in reducing error rates[7-8].

The rest of the paper is organized as follows. In Section 2 we study the signals and system model for IEEE UWB channel modelling. Section 3 is devoted principles of equalizations and receiver structure. In section 4 we study performance analysis for Rake-MMSE receiver. Simulation results are discussed in Section 5. Section 6 concludes the paper.

\section{SIGNALS AND SYSTEM MODEL}

For a single user system, the continuous transmitted data stream is written

$$
A(t)=\sum_{k=-\infty}^{+\infty} d(k) \cdot p\left(t-k \cdot T_{s}\right)
$$

Where $d(k)$ are stationary uncorrelated BPSK data and $T_{s}$ is the symbol duration. Throughout this paper we consider the application of a root raised cosine (RRC) transmit filter $p(t)$ with rolloff factor $\alpha=0.3$. The UWB pulse $p(t)$ has duration $T_{u w b}\left(T_{u w b}<T_{s}\right)$.

The channel models used in this paper are the model proposed by IEEE 802.15.3a Study Group [10]. In the normalized models provided by IEEE 802.15.3a Study Group, different channel characteristics are put together under four channel model scenarios having rms delay spreads ranging from 5 to 26 nsec. For this paper four channel models, derived from the IEEE 802.15 channel modelling working group. In IEEE 802.15.3a working group, the UWB channel is further classified into four models. Channel model 1 (CM1) represents LOS and distance from 0 to $4 \mathrm{~m}$ UWB channel, while channel model 2 (CM2) represents NLOS and distance from 0 to 4 $\mathrm{m}$ UWB channel. Distance from $4 \mathrm{~m}$ to $10 \mathrm{~m}$ and NLOS UWB channel is modelled as CM3 and distance over $10 \mathrm{~m}$ NLOS UWB channels are all classified into the extreme model CM4.

The impulse response can be written as

$$
h(t)=\sum_{p=0}^{M} h_{p} . \delta\left(t-\tau_{p}\right)
$$

Parameter $M$ is the total number of paths in the channel. 


\section{PRINCIPLE OF RECEIVERS STRUCTURE}

\subsection{RAKE RECEIVER}

Rake receivers are used in time-hopping impulse radio systems and direct sequence spread spectrum systems (DS-SS) for matched filtering of the received signal $[9,12]$. The receiver structure consists of a matched filter that is matched to the transmitted waveform that represents one symbol, and a tapped delay line that matches the channel impulse response [11]. It is also possible to implement this structure as a number of correlators that are sampled at the delays related to specific number of multipath components; each of those correlators can be called "Rake finger." A Rake receiver structure is shown in Fig.1.

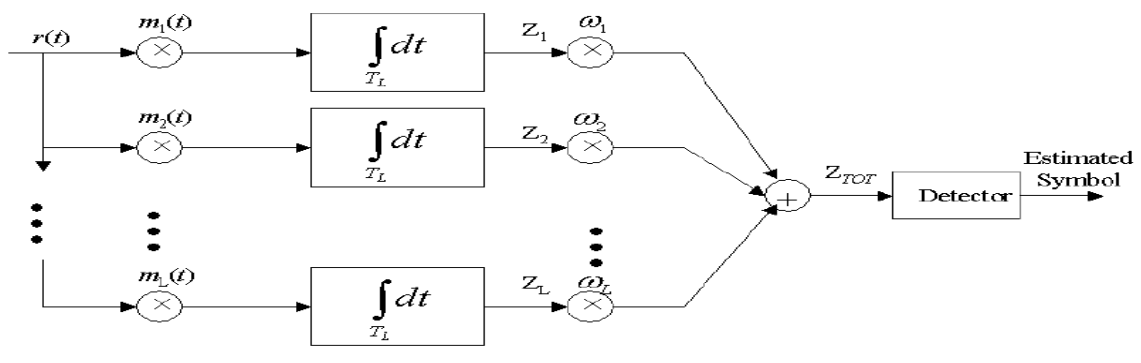

Figure.1. UWB RAKE receiver structure

The received signal first passes through the receiver filter matched to the transmitted pulse and is given by

$$
\begin{aligned}
r(t) & =A(t) * h(t) * p(-t)+n(t) * p(-t) \\
& =\sum_{k=-\infty}^{+\infty} d(k) \sum_{i} h_{i} \cdot m\left(t-k \cdot T_{s}-\tau_{i}\right)+\hat{n}(t)
\end{aligned}
$$

Where $p(-t)$ represents the receiver matched filter, "*”" stands for convolution operation and $n(t)$ is the additive white Gaussian noise (AWGN) with zero mean and variance $N_{0} / 2$. Also, $m(t)=p(t) * p(-t)$ and $n(t)=n(t) * p(-t)$.

Combining the channel impulse response (CIR) with the transmitter pulse shape and the matched filter, we have

$$
\tilde{h}(t)=p(t) * h(t) * p(-t)=\sum_{i=0}^{M} h_{i} \cdot m\left(t-\tau_{i}\right)
$$

The output of the receiver filter is sampled at each Rake finger. The minimum Rake finger separation is $T_{m}=T_{s} / N_{u}$, where $N_{u}$ is chosen as the largest integer value that would result in $T_{m}$ spaced uncorrelated noise samples at the Rake fingers

$$
v\left(n \cdot T_{s}+\tau_{l}^{\prime}+t_{0}\right)=\sum_{k=-\infty}^{+\infty} \tilde{h}\left((n-k) \cdot T_{s}+\tau_{i}^{\prime}+t_{0}\right) \cdot d(k)
$$

where $\tau_{l}{ }^{\prime}$ is the delay time corresponding to the $l^{\text {th }}$ Rake finger and is an integer multiple of $T_{m}$. Parameter $t_{0}$ corresponds to a time offset and is used to obtain the best sampling time. Without loss of generality, $t_{0}$ will be set to zero in the following analysis.

\subsection{MMSE STRUCTURE}

In reality the noise component due to the physical channel cannot be ignored. In the presence of additive Gaussian noise at the receiver input, the output of the equalizer at the $\mathrm{n}^{\text {th }}$ sampling instant is given by 


$$
\hat{y}_{n}=\sum_{K=-N}^{N} b_{k} r_{n-k}
$$

The mean square error (MSE) for the equalizer having $2 N+1$ taps, denoted by $J(N)$ is

$$
J(N)=E\left|x_{n}-\hat{y}_{n}\right|^{2}=E\left[\left(x_{n}-\sum_{k=-N}^{N} b_{k} r_{n-k}\right)^{2}\right]
$$

$J(N)$ with respect to the equalizer coefficients $\left(b_{k}\right)$ is obtained by the following differentiation:

$$
\frac{d J(N)}{d b_{k}}=0
$$

Equation (7) leads to the necessary condition for the minimum MSE given by

$$
R_{r} b=R_{x r} \quad b=R_{r}^{-1} R_{x r}
$$

WHERE

$$
\begin{aligned}
& R_{x r}=\left(R_{x r}(-N) \ldots R_{x r}(0) \ldots R_{x r}(N)\right)^{T} \\
& R_{r}=\left[\begin{array}{lll}
R_{r}(0) \ldots \ldots . & R_{r}(N) \ldots \ldots . & R_{r}(2 N) \\
\cdot & \cdot & \cdot \\
\cdot & \cdot & \cdot \\
R_{r}(-2 N+1) & R_{r}(-N+1) & R_{r}(1) \\
R_{r}(-2 N)_{2 N} & R_{r}(-N) & R_{r}(0)
\end{array}\right]
\end{aligned}
$$

\subsection{RAKE-MMSE STRUCTURE}

The receiver structure is illustrated in Fig. 2 and consists in a Rake receiver followed by a linear equalizer. As we will see later on, a structure gives better performances over UWB channels when the number of equalizer taps is sufficiently large. The received signal first passes through the receiver filter matched to the transmitted pulse (3). The output of the receiver filter is sampled at each Rake finger. The minimum Rake finger separation is $T_{m}=T_{s} / N_{u}$, where $N_{u}$ is chosen as the largest integer value that would result in Tm spaced uncorrelated noise samples at the Rake fingers(4). In a first approach, complete channel state information (CSI) is assumed to be available at the receiver. For general selection combining, the Rake fingers $(\beta$ 's) are selected as the largest $L\left(L \leq N_{u}\right)$ sampled signal at

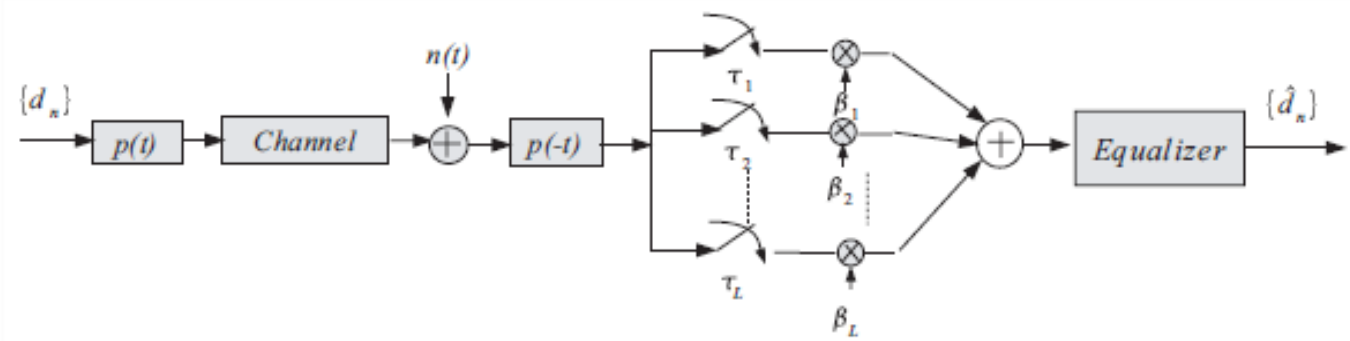

Figure.2. UWB RAKE-MMSE receiver structure

the matched filter output within one symbol time period at time instants $\tau_{l}{ }^{\prime}, l=1,2, \ldots, L$. In fact, since a UWB signal has a very wide bandwidth, a Rake receiver combining all the paths of the incoming signal is practically unfeasible. This kind of Rake receiver is usually named a ARake receiver. A feasible implementation of multipath diversity combining can be obtained by 
a selective-Rake (SRake) receiver, which combines the $L$ best, out of $N_{u}$, multipath components. Those $\mathrm{L}$ best components are determined by a finger selection algorithm. For a maximal ratio combining (MRC) Rake receiver, the paths with highest signal-to-noise ratios (SNRs) are selected, which is an optimal scheme in the absence of interfering users and intersymbol interference (ISI). For a minimum mean square error (MMSE) Rake receiver, the "conventional" finger selection algorithm is to choose the paths with highest signal-tointerference-plus-noise ratios (SINRs) [2]. Our case doesn't deal with multiuser UWB communication but we study channels with high delay dispersion, so the first criterion $(L$ highest SNR's) can be chosen. The noiseless received signal sampled at the $l^{\text {th }}$ Rake finger in the $n^{\text {th }}$ data symbol interval is given by equation (5). The Rake combiner output at time $t=n \cdot T_{s}$ is

$$
y[n]=\sum_{l=1}^{L} \beta_{l} \cdot v\left(n \cdot T_{s}+\tau_{l}^{\prime}\right)+\sum_{l=1}^{L} \beta_{l} \cdot \hat{n}\left(n \cdot T_{s}+\tau_{l}^{\prime}\right)
$$

Choosing the correct Rake finger placement leads to the reduction of ISI and the performance can be dramatically improved when using an equalizer to combat the remaining ISI. Considering the necessary tradeoff between complexity and performance, a sub-optimum classical criterion for updating the equalizer taps is the MMSE criterion.

\section{PERFORMANCE ANALYSIS}

In this part, due to the lack of place we will only discuss the matrix block computation of linear equalizers. Furthermore, we suppose perfect channel state information (CSI). Assuming that the $\mathrm{n}$ data bit is being detected, the MMSE criterion consists in minimizing

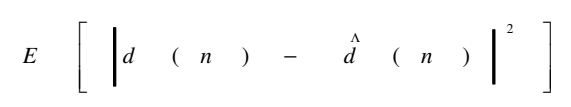

where $d(n)$ is the equalizer output. Rewriting the Rake output signal, one can distinguish the desired signal, the undesired ISI and the noise as

$$
\begin{aligned}
y(n)=\left[\sum_{l=1}^{L} \beta_{l} \cdot \hat{h}\left(\tau_{l}^{\prime}\right)\right] \cdot d(n)+ & \sum_{k \neq n} \sum_{l=1}^{L} \beta_{l} \cdot \hat{h}\left((n-k) \cdot T_{s}+\tau_{l}^{\prime}\right) \cdot d(k) \\
& +\sum_{l=1}^{L} \beta_{l} \cdot \hat{n}\left(n \cdot T_{s}+\tau_{l}^{\prime}\right)
\end{aligned}
$$

where the first term is the desired output. The noise samples at different fingers, $n\left(n . T s+\tau_{l}{ }^{\prime}\right)$, $l=1 \ldots L$, are uncorrelated and therefore independent, since the samples are taken at approximately the multiples of the inverse of the matched filter bandwidth. It is assumed that the channel has a length of $\left(n_{1}+n_{2}+1\right) \cdot T_{s}$. That is, there is pre-cursor ISI from the subsequent $n_{1}$ symbols and post-cursor ISI from the previous $n_{2}$ symbols, and $n_{1}$ and $n_{2}$ are chosen large enough to include the majority of the ISI effect. Using (8), the Rake output can be expressed now in a simple form as

$$
y(n)=\alpha_{0} . d(n)+\sum_{\substack{k \neq-n_{1} \\ k \neq 0}}^{n} \alpha_{k} \cdot d(n-k)+\dot{n}(n)
$$

$=\phi^{T}[n]+\dot{n}(n)$
where coefficient $\alpha_{K}$ 's are obtained by matching (14) and (15).
$\phi=\left[\alpha_{n_{1}} \ldots \alpha_{0} \ldots \alpha_{n_{2}}\right]$ and $d[n]=\left[d\left(n+n_{1}\right) \ldots d(n) . \quad \ldots d\left(n-n_{2}\right)\right]^{T}$

The superscript denotes the transpose operation. The output of the linear equalizer is obtained as 


$$
\hat{d}(n)=\sum_{r=-k_{1}}^{k_{2}} c_{r} \cdot y(n-r)=c^{T} \gamma(n)+c^{T} \eta(n)
$$

where $c=\left[c_{-} \ldots c_{1} \ldots c_{0} \ldots \kappa_{2}\right]$ contains the equalizer taps. Also

$$
\begin{aligned}
& \gamma[n]=\left[\phi^{T} d\left[n+K_{1}\right] \ldots \phi^{T} d[n] \ldots \phi^{T} d\left[n-K_{2}\right]\right]^{T} \\
& \eta[n]=\left[\tilde{n}\left(n+K_{1}\right) \ldots \tilde{n}(n) \ldots \tilde{n}\left(n=K_{2}\right)\right]^{T}
\end{aligned}
$$

The mean square error (MSE) of the equalizer,

$$
E\left[\left|d(n)-c^{T} \gamma[n]-c^{T} \eta[n]\right|^{2}\right]
$$

which is a quadratic function of the vector $c$, has a unique minimum solution. Here, the expectation is taken with respect to the data symbols and the noise. Defining matrices $R, p$ and $N$ as

$$
\begin{aligned}
& \mathbf{R}=\mathrm{E}\left[\gamma[\mathrm{n}] \cdot \gamma^{\mathrm{T}}[\mathrm{n}]\right] \\
& \mathbf{p}=\mathrm{E}[\mathrm{d}(\mathrm{n}) \cdot \gamma[\mathrm{n}]] \\
& \mathbf{N}=\mathrm{E}\left[\eta[\mathrm{n}] \cdot \eta^{\mathrm{T}}[\mathrm{n}]\right]
\end{aligned}
$$

The equalizer taps are given by

$$
c=(R+N)^{-1} \cdot p
$$

and the MMSE is

$$
\begin{aligned}
& J_{\text {min }}=\sigma_{d}^{2}-p^{T}(R+N)^{-1} \cdot p \\
& \sigma_{d}^{2}=E\left[|d(n)|^{2}\right]
\end{aligned}
$$

Evaluating the expectation over $R$ and $p$ with respect to the data and the noise, we have

$$
p=\left[\alpha_{K} \ldots \alpha_{0} \ldots \alpha_{-K_{2}}\right]^{T} \quad R=\left[r_{i, j}\right]_{K_{1}+K_{2}+1, K_{1}+K_{2}+1}
$$

Where

$$
\begin{gathered}
\mathrm{r}_{\mathrm{i}, \mathrm{j}}=\phi^{\mathrm{T}} \mathrm{F}_{\mathrm{i}, \mathrm{j}} \phi \text { and } \mathrm{F}_{\mathrm{i}, \mathrm{j}}=\left[\mathrm{f}_{1, \mathrm{k}}\right]_{\mathrm{n}_{1}+\mathrm{n}_{2}+1, \mathrm{n}_{1}+\mathrm{n}_{2}+1} \\
f_{l k}=\left(\begin{array}{ll}
1, & l-k=j-i \\
0, & l-k \neq j-i
\end{array}\right. \\
\mathrm{N}=\mathrm{E}\left[\eta[\mathrm{n}] \eta^{\mathrm{T}}[\mathrm{n}]\right]=\frac{\mathrm{N}_{0}}{2} \cdot\left(\sum_{\mathrm{l}=1}^{\mathrm{L}} \beta_{1}^{2}\right) \cdot \mathrm{I}_{\mathrm{K}_{1}+\mathrm{K}_{2}+1}
\end{gathered}
$$

Where $I$ is the identity matrix. This Rake-equalizer receiver will eliminate ISI as far as the number of equalizer's taps gives the degree of freedom required. In general, the equalizer output can be expressed as

$$
\stackrel{\Lambda}{d}(n)=q_{0} \cdot d(n)+\sum_{i=0} q_{1} \cdot d(n-i)+w(n)
$$


with $\quad q_{n}=\alpha_{n} \cdot c_{n}$

The variance of $w(n)$ is

$$
\sigma_{w(n)}^{2}=\left(\sum_{i=-K_{1}}^{K_{2}} c_{i}^{2}\right)\left(\sum_{l=1}^{L} \beta_{1}^{2}\right) \cdot E_{p} \cdot N_{0} / 2
$$

Where $E_{p}$ is the pulse energy.

Where matrix 0 is the all zero matrix.

The MMSE feedback taps are then obtained in terms of feed forward taps and matrix $U$.

$$
\left[c_{1} \ldots \ldots \ldots \ldots c_{k 2]}=\left[c-{ }_{K 1} \ldots \ldots \ldots \ldots c_{0}\right] U^{T}\right.
$$

Conditioned on a particular channel realization, $h=\left[h_{1} \ldots \ldots . . h_{I}\right]$, an upper bound for the probability of error using the chernoff bound technique given by

$$
P\left(\hat{d}_{n} \neq d_{n} \mid h\right) \leq \exp \left(-\frac{1-J_{\min } / \sigma_{d}^{2}}{2 J_{\min }}\right)
$$

An exact BER expression with independent noise and ISI terms can be expressed as a series expansion is given by

$$
P\left(\hat{d}_{n} \neq d_{n} \mid h\right)=\frac{1}{2}-\frac{2}{\pi} \sum_{\substack{z=1 \\ z o d d}}^{z} \frac{\exp \left(-z^{2} w^{2} / 2\right) \sin \left(z w q_{0}\right)}{z} \times \prod_{\substack{n=N_{1} \\ n \neq 0}}^{N_{2}} \cos \left(z w q_{n}\right)
$$

Note that ISI comes from the interfering symbols in the range of $N_{l} T_{s}$ and $N_{2} T_{s}$. Parameter $\mathrm{z}$ and $\mathrm{w}$ determine the accuracy of the error rate given by (30).

we can simply set the $q_{i}^{\prime}$ s that are within the span of the feedback taps to be 0 , which corresponds to zero post-cursor ISI for the span of feedback taps.

\section{SIMULATION STUDY AND ANALYSIS}

\subsection{Signal Waveform}

The pulse shape adopted in the numerical calculations and simulations is the second derivative of the Gaussian pulse given by

$$
\mathrm{w}(\mathrm{t})=\left[1-4 \pi(\mathrm{t} / \varepsilon)^{2}\right] \exp \left(-2 \pi(\mathrm{t} / \varepsilon)^{2}\right)
$$

The pulse waveform and power spectral density are showed as figure 3 .
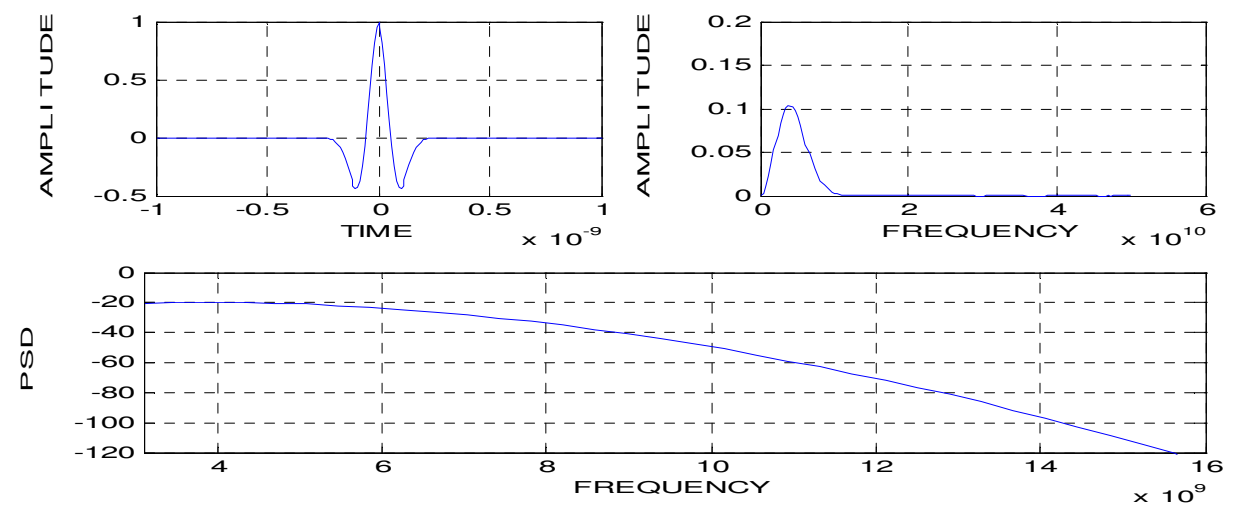

Figure.3. Second derivative of Gaussian pulse 


\subsection{Channel Model Parameter (IEEE 802.15.3a)}

As we mentioned it before, we study the case of UWB channels CM1, CM2, CM3 and CM4 channel models [10]. We have used an oversampling factor of eight for the root raised cosine (RRC) pulse. According to this sampling rate, time channel spread is chosen equal to 100 for CM4 and 70 for CM3, this corresponds to respectively $12=100 / 8$ and $9=70 / 8$ transmitted symbols. This choice enables to gather $99 \%$ of the channel energy. The coherence bandwidths of CM1, CM2, CM3, and CM4 simulation are $27 \mathrm{MHz}, 26 \mathrm{MHz}, 10.6 \mathrm{MHz}$, and $5.9 \mathrm{MHz}$ respectively. The data rate is chosen to be $200 \mathrm{Mbps}$, one of the optional data rates proposed for IEEE standard. The size of the transmitted packets is equal to 2560 BPSK symbols including a training sequence of length 512. CIR remains constant over the time duration of a packet. The root raised cosine (RRC) pulse with roll off factor $\alpha=0.5$ is employed as the pulse-shaping filter. The CM1-CM4 indoor channel model is adopted in simulation. The simulated channel impulse responses for CM1, CM2, CM3 and CM4 are shown in figure 4, figure 6, figure 8 and figure 10. The power delay profiles for CM1, CM2, CM3 and CM4 are plotted in figure 5, 7, 9 and 11 respectively. The simulation parameter settings for the entire four channel models are listed in Table.1.

Table.1 Parameter Settings for IEEE UWB Channel Models

\begin{tabular}{|l|l|l|l|l|l|l|c|}
\hline Scenario & $\Lambda(1 / \mathrm{ns})$ & $\lambda(1 / \mathrm{ns})$ & $\Gamma(1 / \mathrm{ns})$ & $\gamma(1 / \mathrm{ns})$ & $\sigma_{\xi}(\mathrm{dB})$ & $\sigma_{\varsigma}(\mathrm{dB})$ & $\sigma_{\mathrm{g}}(\mathrm{dB})$ \\
\hline CM1 & 0.0233 & 2.5 & 7.1 & 4.3 & 3.3941 & 3.3941 & 3 \\
\hline CM2 & 0.04 & 0.5 & 5.5 & 6.7 & 3.3941 & 3.3941 & 3 \\
\hline CM3 & 0.0067 & 2.1 & 14 & 7.9 & 3.3941 & 3.3941 & 3 \\
\hline CM4 & 0.0067 & 2.1 & 24 & 12 & 3.3941 & 3.3941 & 3 \\
\hline
\end{tabular}

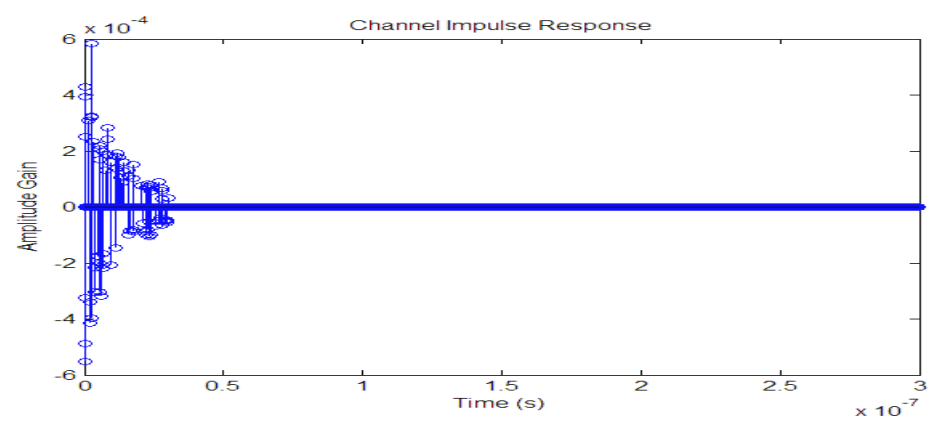

Figure.4. Channel Impulse Response of CM1 (LOS)

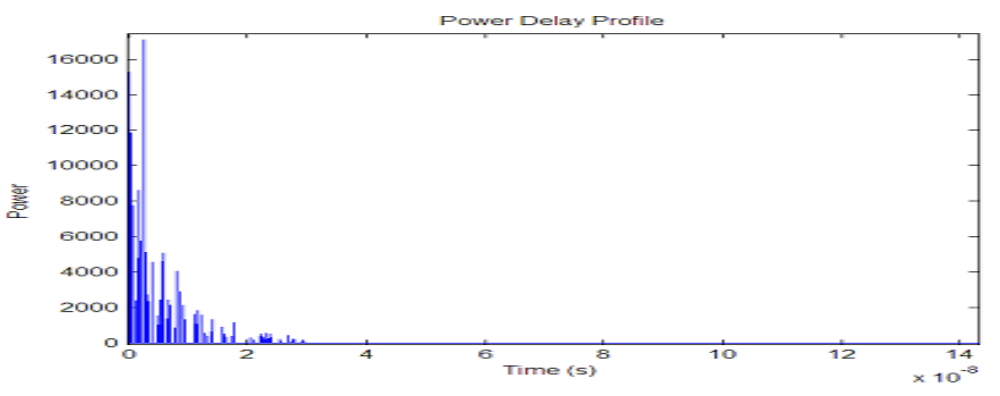

Figure.5. Power Delay Profile of CM1 Channel 


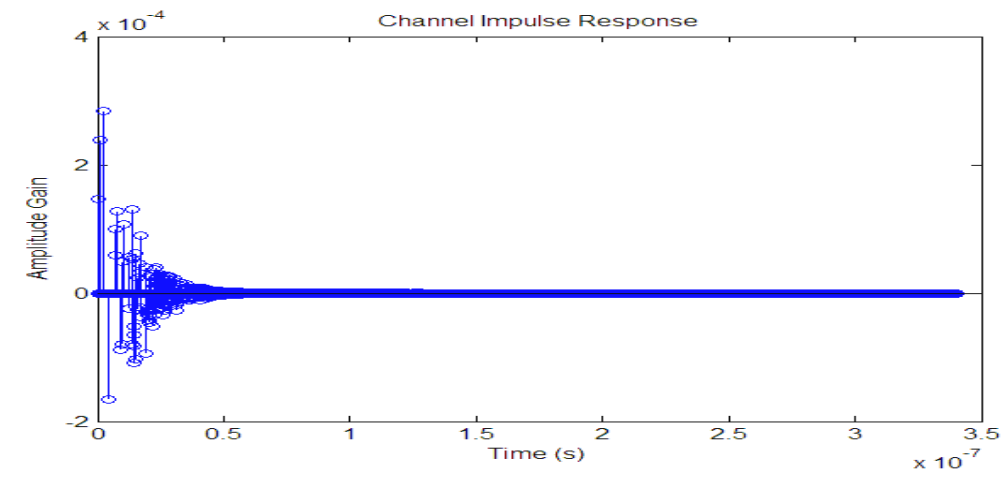

Figure.6. Channel Impulse Response of CM2 (NLOS)

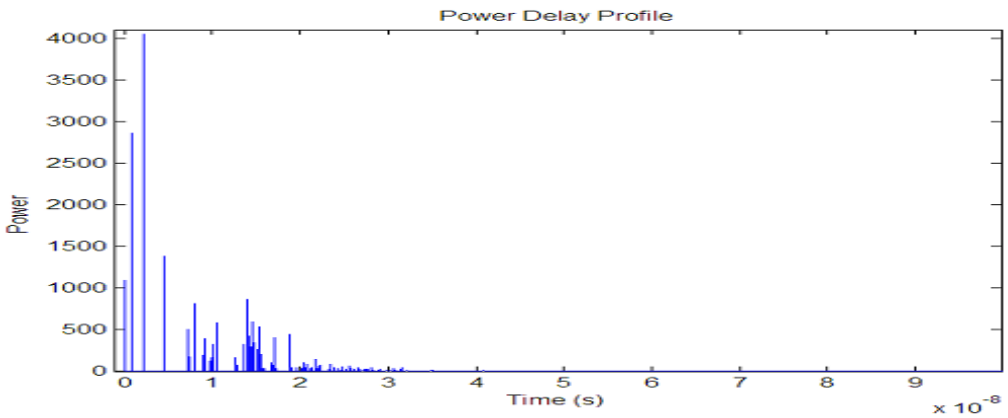

Figure.7. Power Delay Profile of CM2 Channel

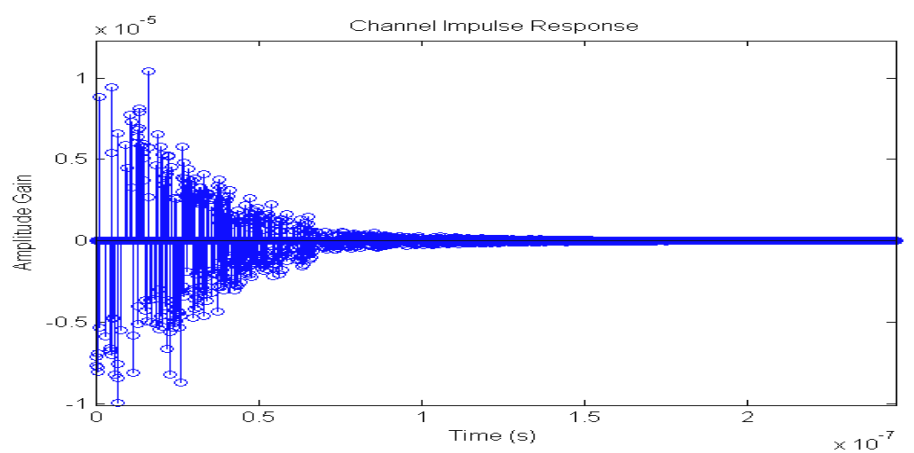

Figure.8. Channel Impulse Response of CM3 (NLOS)

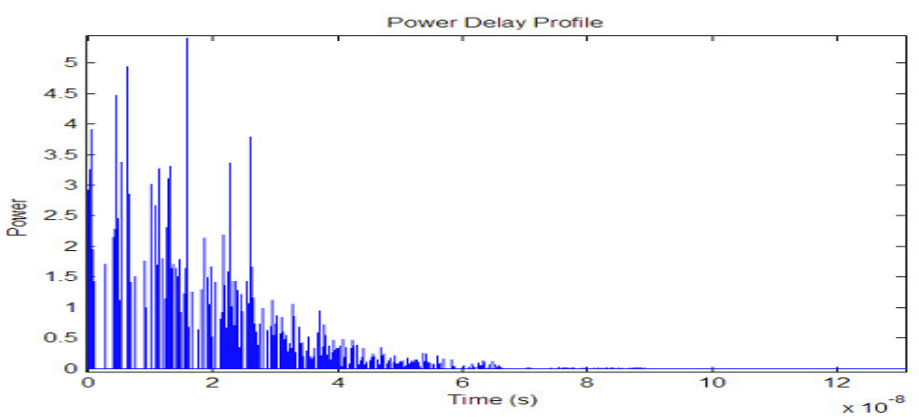

Figure.9. Power Delay Profile of CM3 Channel 


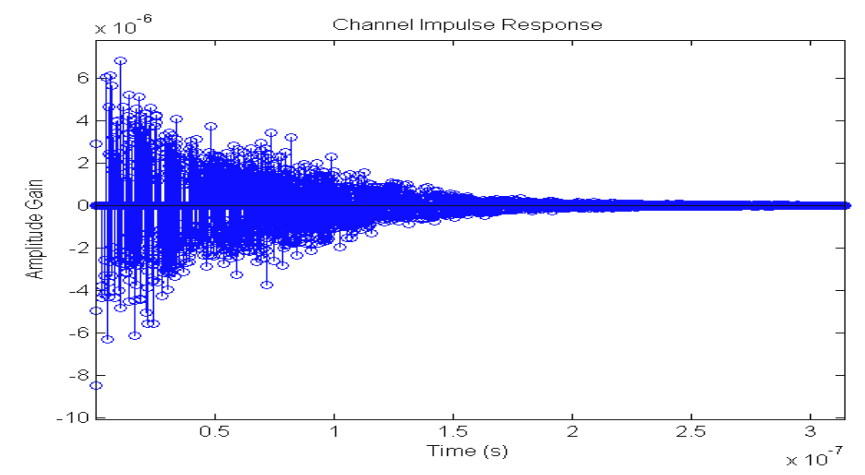

Figure.10. Channel Impulse Response of CM4 (NLOS)

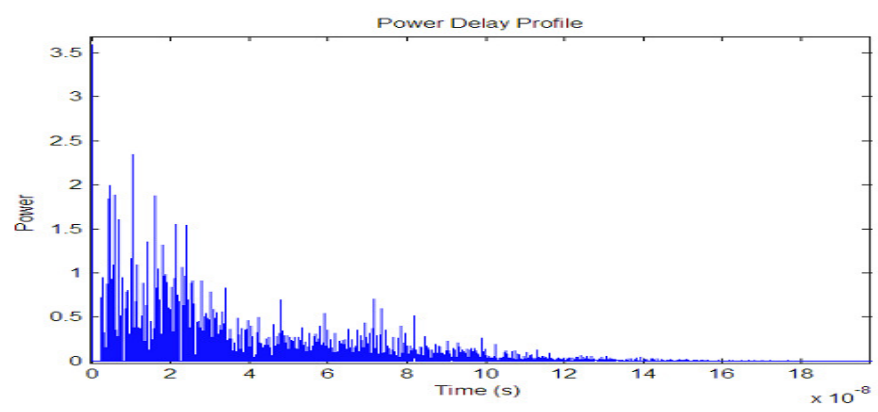

Figure.11. Power Delay Profile of CM4 Channel

\subsection{BER ANALYSIS}

In the case of time domain equalization, we have at first to optimize the number of Rake fingers and the number of equalizer taps. The Rake fingers are regularly positioned according to time channel spread and the number of fingers. Figure12, figure 13, figure 14 and figure 15 shows the effect rake, mmse and rake-mmse using Monte-Carlo simulation. The Rake combiner output at time $t=n . T_{s}$. As expected, using a 5 taps MMSE equalizer to compensate for ISI, a relative improvement is observed. The major comparison lies in the rake-mmse receiver having 5 tap, 10 rake fingers versus the Rake receiver with 10 rake fingers. Table. 2 provides the simulation parameter.

Table.2 Simulation Parameter

\begin{tabular}{|l|c|}
\hline \multicolumn{1}{|c|}{ Parameter } & values \\
\hline Data rate & $200 \mathrm{Mbps}$ \\
\hline Pulse width & 0.18 \\
\hline Symbol duration & $5 \mathrm{~ns}$ \\
\hline Number of equalizer taps & 5 \\
\hline Number of Rake fingers & 10 \\
\hline Pulse energy & 1 \\
\hline $\mathrm{T}_{\mathrm{m}}$ & $0.1786 \mathrm{~ns}$ \\
\hline \multicolumn{1}{|c|}{$\mathrm{N}_{\mathrm{u}}$} & 28 \\
\hline Channel spread & 100 \\
\hline Pilot carrier & 500 \\
\hline
\end{tabular}


Rake-mmse receiver has around $3 \mathrm{~dB}$ performance improvement compared to a rake receiver for CM1 channel model as shown in figure 12 . Where at $10^{-3}$ BER floor, Rake-mmse receiver has around 1.6dB SNR performance improvement compared to a channel with rake receiver for CM2 channel model as shown in figure 13.

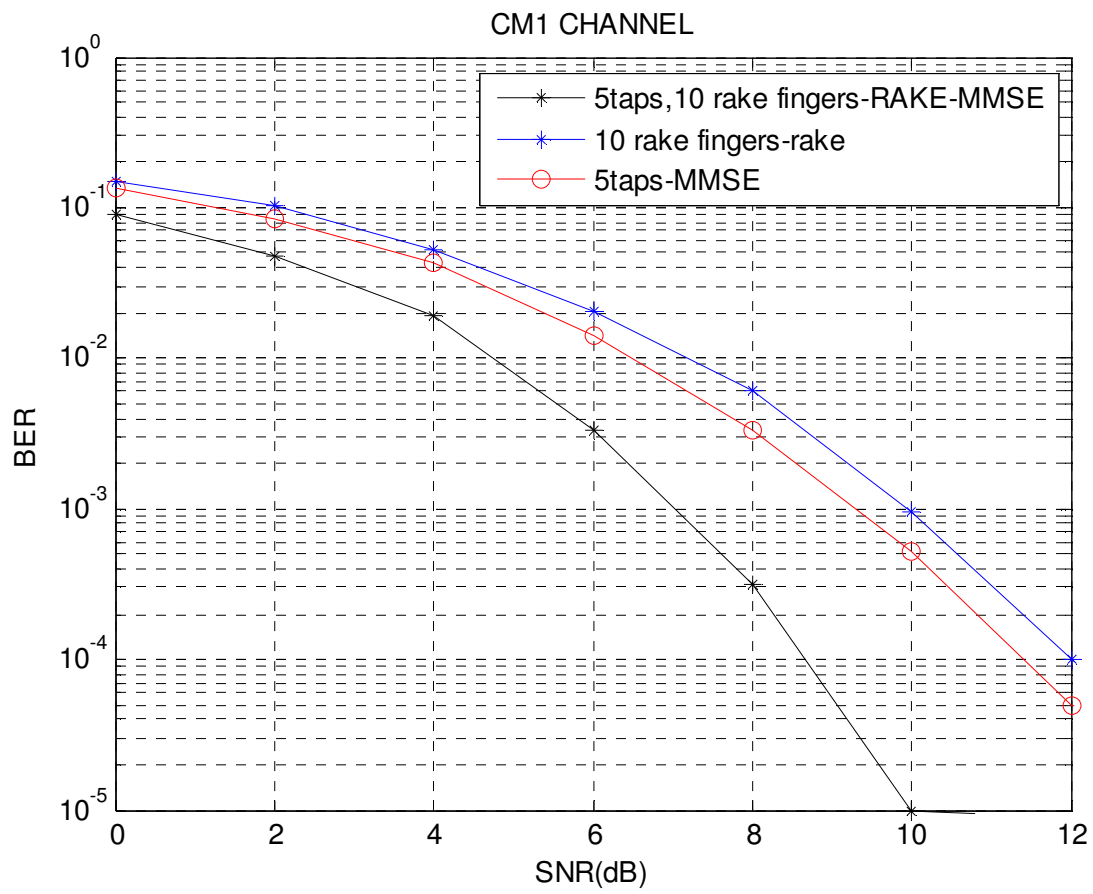

Figure.12. Performance of UWB receivers for CM1 channel model

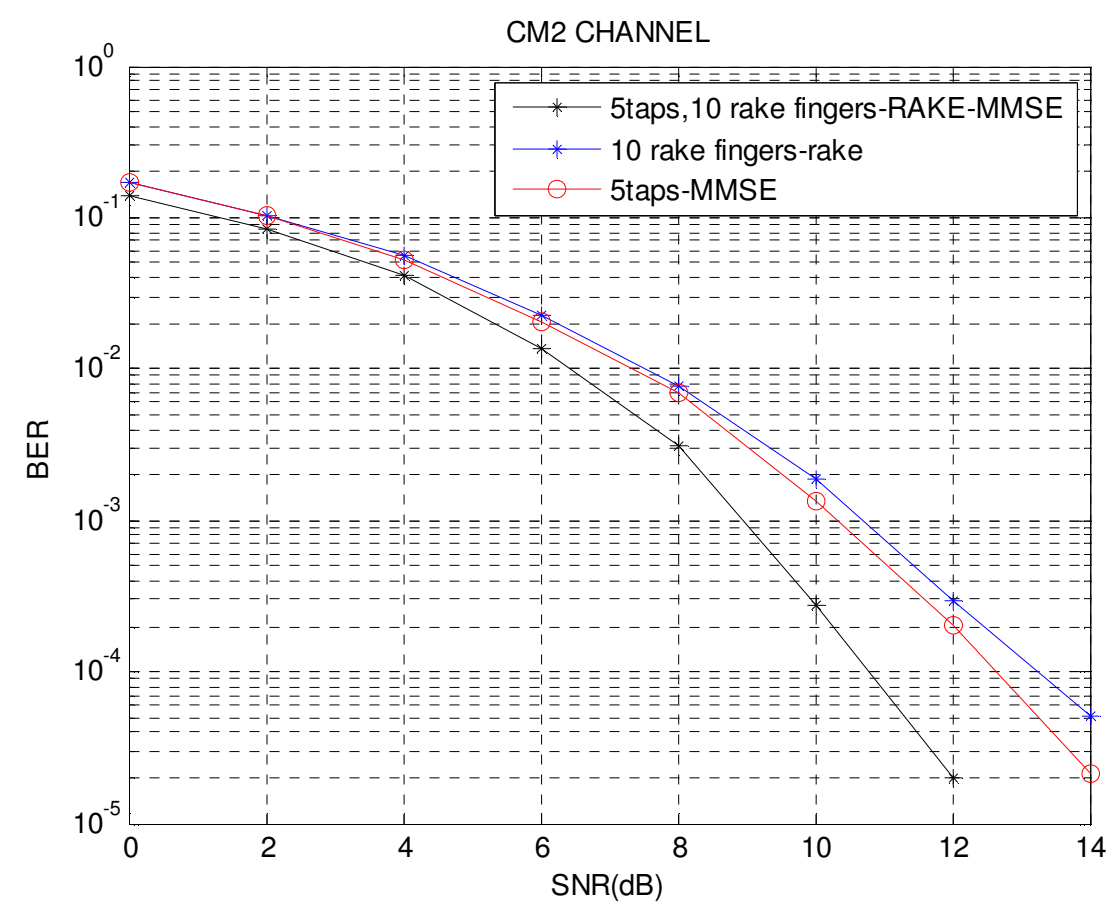

Figure.13. Performance of UWB receivers for CM2 channel model 


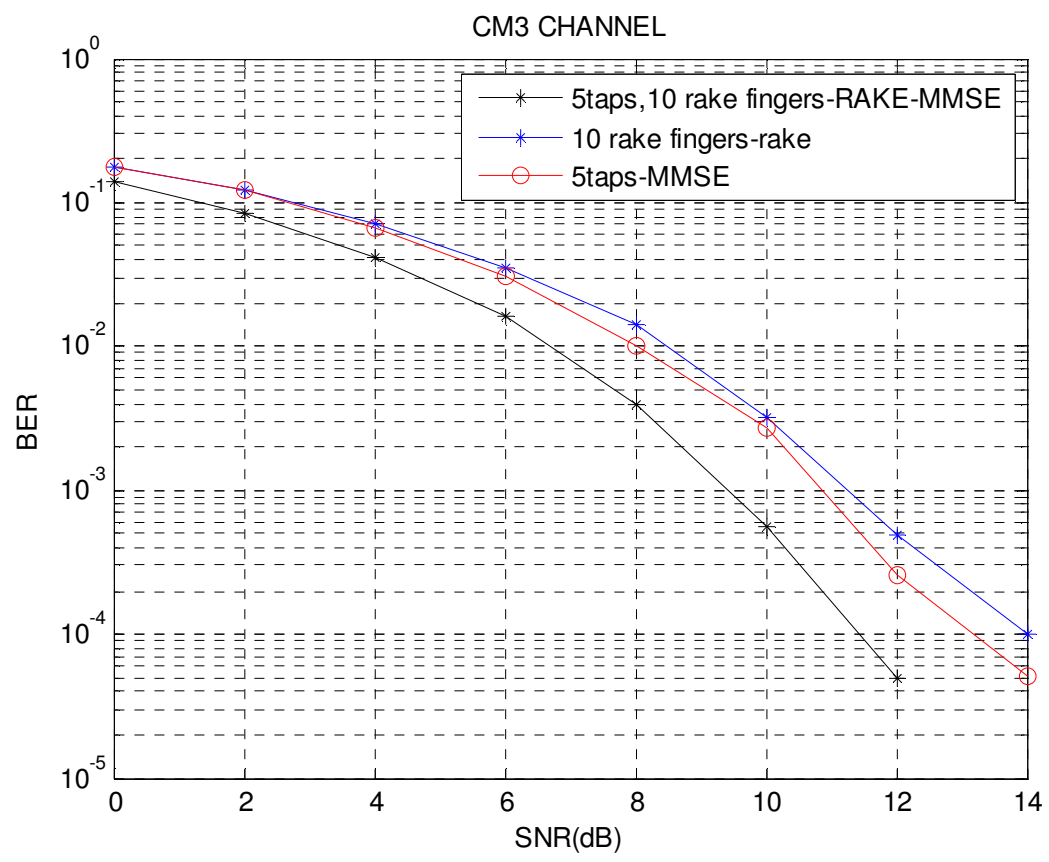

Figure.14. Performance of UWB receivers for CM3 channel model

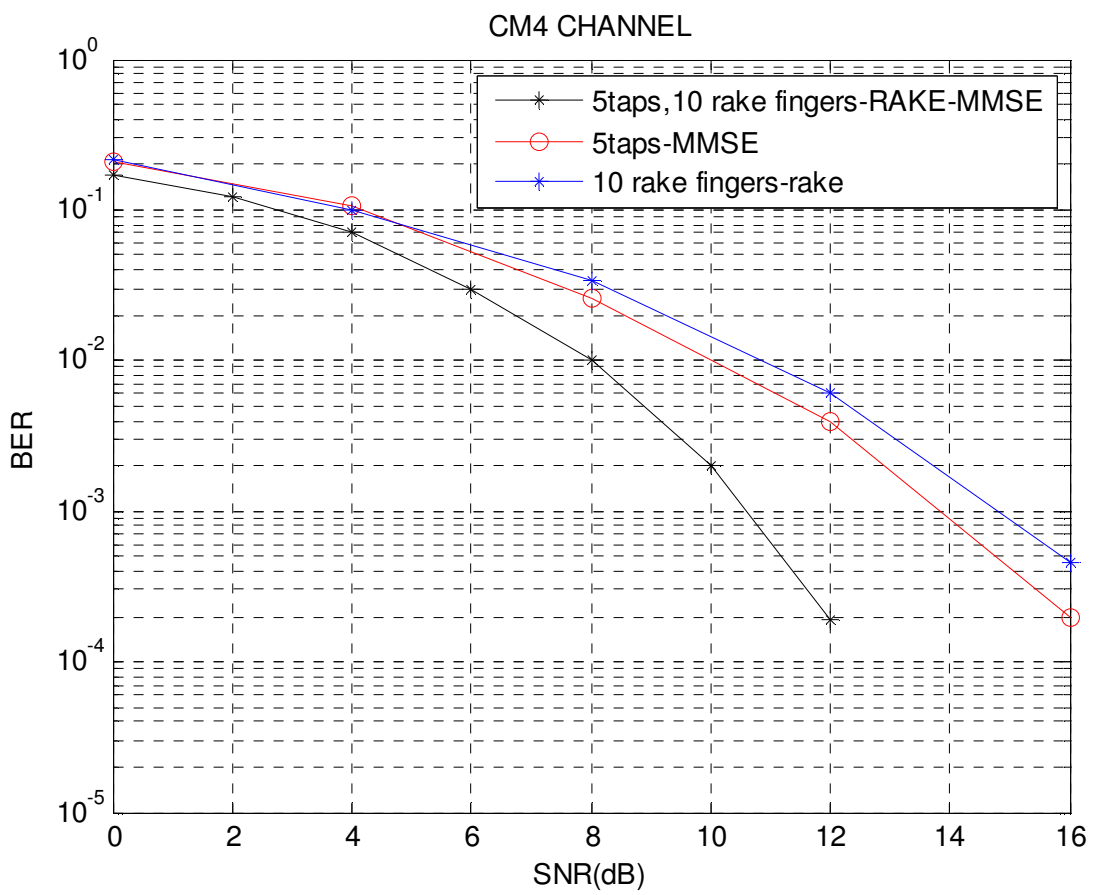

Figure.15. Performance of UWB receivers for CM4 channel model

Rake-mmse receiver has around1.8dB performance improvement compared to a channel with rake receivers for CM3 channel model as shown in figure 14. Using CM4 channel data, for same parameter the simulation results obtained as shown in figure 15.Using RakeMMSE, i.e. rake-mmse and a rake receiver a gain of around $4 \mathrm{~dB}$ is observed. This result can be explained by considering the fact that at high SNR's it is mainly the ISI that 
affects the system performance whereas at low SNR's the system noise is also a major contribution in system degradation (more signal energy capture is required). The performance dramatically improves when the number of Rake fingers and the equalizer taps are increased simultaneously in Rake-MMSE receiver. As expected the receiver has better performance over CM3 with smaller delay spread than CM4 on non-line of sight (NLOS) indoor channel models.

\section{CONCLUSION}

The receivers combats inter-symbol interference by taking advantage of the Rake and equalizer structure by using different UWB channel models CM1-CM4 for high data rate WPAN system. For a MMSE equalizer operating at low to medium SNR's, the number of Rake fingers is the dominant factor to improve system performance, while, at high SNR's the number of equalizer taps plays a more significant role in reducing error rates. The IEEE 802.15a (TG3a) standard which is to provide a low complexity, low cost, low power consumption and high data-rate among Wireless Personal Area Network (WPAN) devices. One can observe at BER floor having high SNR values the receiver has better performance over CM1 (LOS) with smaller delay spread than CM2 (NLOS) channel models. Rake-mmse receiver has around1.8dB performance improvement in CM3 (NLOS) channel model compared to CM4 (NLOS) channel model. This architecture has opened up new directions in designing efficient adaptive equalizers and can be implemented in DSP processors for real - time applications.

\section{REFERENCES}

[1] A. Rajeswaran, V.S. Somayazulu and J. Foerster, "Rake performance for a pulse based UWB system in a realistic UWB indoor channel", IEEE ICC'03., vol. 4, pp. 2879-2883, May 2003.

[2] S. Gezici, H. V. Poor and H. Kobayashi, "Optimal and Suboptimal Finger Selection Algorithms for MMSE Rake Receivers in Impulse Radio Ultra-Wideband Systems", in Proc IEEE WCNC 2005, New-Orleans, Mar. 2005.

[3] J. R. Foerster, "The effect of multipath interference on the performance of UWB systems in an indoor wireless channel", in Proc. 2001 Spring Vehicular Technology Conf., pp. 1176-1180, May 2001.

[4] J. D. Choi and W. E. Stark, "Performance of ultra-wideband communications with suboptimal receivers in multipath channels", IEEE J. Select. Areas Communication, vol. 20, pp. 1754-1766, Dec. 2002.

[5] D. Cassioli, M. Z. Win, F. vAtalaro and F. Molisch, "Performance of low-complexity RAKE reception in a Realistic UWB channel”, in Proc. Int. Conf. Communication (ICC), vol. 2, May 2002, pp. 763-767.

[6] T. Strohmer, M. Emani, J. Hansen, G. Papanicolaou and A. J. Paulraj, "Application of TimeReversal with MMSE Equalizer to UWB Communications", in Proc. IEEE Globecom'04., vol. 5, pp. 3123-3127, Dec. 2004.

[7] B. Mielczarek, M. O. Wessman and A. Svensson, "Performance of Coherent UWB Rake Receivers With Channel Estimators", in Proc. Vehicular Technology Conf. (VTC), vol .3, Oct. 2003, pp. 1880-1884.

[8] A. Rajeswaran, V. S. Somayazulu and J. R. Forester, "Rake Performance for a Pulse Based UWB System in a Realistic UWB Indoor Channel”, in Proc. IEEE Int. Conf. Communication (ICC), vol. 4, May 2003, pp. 2829-2833. 
[9] Y. Ishyama and T. Ohtsuki, "Performance Evaluation of UWB- IR and DS-UWB with MMSEfrequency Domain Equalization (FDE)", in Proc. IEEE Global Telecommunication Conf. (Globecom), vol. 5, Nov-dec 2004, pp. 3093-3097.

[10] J.F. Foerster, M. Pendergrass and A. F. Molisch, "A Channel Model for Ultrawideband Indoor Communication", MERL (Mitsubishi Electric Research Laboratory) Report TR-2003-73, Nov. 2003.

[11] P. Balaban and J. Salz, "Optimum diversity combining and equalization in digital data transmission with applications to cellular mobile radio-part I: theoretical considerations," IEEE Trans. Communication, vol. 40, pp. 885-894, May 1992.

[12] M.Z. Win, R.A Scholtz, "Ultra-wide bandwidth time- hopping spread spectrum impulse radio for wireless multiple-access communications", IEEE Trans. Communication, pp. 679-691, vol. 48,no. 4, April 2000.

\section{Authors}

Dr.Susmita Das, Ph.D, is Associate Professor of Electrical Engineering, NIT, and Rourkela, India. She has twenty years of teaching and research experience and has many research papers to her credit. She is Member IEEE, Fellow IETE, LM ISTE and Member IEI. Her research interests include Wireless Communication, DSP, and Application of Soft Computing Techniques etc.

Bikramaditya Das received his B.Tech in Electronics and Telecommunications Engineering from the University of B.P.U.T, Rourkela, Orissa, India, in 2007. From 2008 to till now he is a research Fellow under the Department of Electrical Engineering at the N.I.T, Rourkela, India. He is a Member IEI.
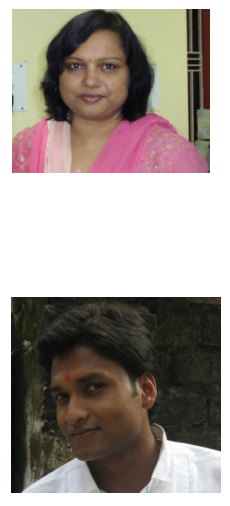